\title{
Characterization of Nanoparticle Binding Dynamics in Microcirculation using an adhesion Probability Function
}

\author{
Salman Sohrabi \\ Department of Mechanical Engineering \& Mechanics \\ Lehigh University, Bethlehem, PA 18015, USA \\ Sas713@Lehigh.edu
}

\section{Doruk Erdem Yunus}

Department of Mechanical Engineering \& Mechanics

Lehigh University, Bethlehem, PA 18015, USA

dey212@lehigh.edu

\section{Jiang Xu}

School of Mechanics and Engineering

Southwest Jiaotong University, Chengdu

xujiang0123@163.com

Jie Yang ${ }^{1}$

School of Mechanics and Engineering

Southwest Jiaotong University, Chengdu

yangchenjie@home.swjtu.edu.cn

\section{Yaling Liu ${ }^{2}$}

Department of Mechanical Engineering \& Mechanics

Bioengineering Program

Lehigh University, Bethlehem, PA 18015, USA

Yal310@lehigh.edu

\footnotetext{
${ }^{1}$ *Corresponding author. Tel.: +86-28-8760-0797 ; fax: +86-28-8760-0797 .

Postal address: School of Mechanics and Engineering, Southwest Jiaotong University, Chengdu 610031, China

E-mail address:yangchenjie@home.swjtu.edu.cn

$2 *$ Corresponding author. Tel.: +1-610-758-5839; fax: +1-610-758-6224

Postal address: 19 Memorial Dr W, Packard Lab, Lehigh University, Bethlehem, PA 18015

E-mail address:yal310@lehigh.edu
} 


\begin{abstract}
Quantitative understanding of nanoparticles transport and adhesion dynamic in microcirculation is very challenging due to complexity of fluid dynamics and imaging setup. In-vitro experiments within microfluidic channels showed the significant influence of shear rate, carrier size, particle-substrate chemistry and vessel geometry on particle deposition rate. However, there is few theoretical models that can accurately predict experimental results. We have developed a numerical model to predict nanoparticle transport and binding dynamics and verified with our previous in-vitro tests results. A binding probability function is used to simplify the carrier attachment and detachment processes. Our results showed that due to the complex dynamics of particle transport and adhesion mechanism, the correlation between binding probability and actual deposition rate is not linear. Using experimental data, it is shown that the binding probability of small particles changes slightly with shear rate whereas the chance of binding for big particles decreases exponentially with shear. Our particulate model also captured some phenomena that cannot be achieved by continuum approach such as accumulation of drug particles in close vicinity of vessel wall. In addition, the effects of channel geometry and antibody density on particle binding are discussed extensively. The results from our particulate approach agrees well with experimental data suggesting that it can be used as a simple, yet efficient predictive tool for studying drug carrier binding in microcirculation.
\end{abstract}

Keywords: Drug delivery, Microfluidic channel, Binding density, Nano-Particle, Binding probability function.

\title{
I. Introduction
}

Nanoparticles have been widely studied as potential multifunctional carrier platforms for therapeutic drug delivery and imaging applications [1-5]. To deliver drugs to target diseased site, carriers of various sizes and shapes are needed to circulate in the body for a sufficient period of time. They need to laterally drift toward the vascular wall, interact with the receptors expressed on the vascular wall, and finally bind at the diseased region. Particle sizes can range from a few tens/hundreds of nanometers, such as dendrimers, micelles, nano-shells and polymeric spheres [6-9], to a few microns, such as lipid and silica-based microspheres $[10,11]$. Furthermore, their shape can also vary from spheroidal to cylindrical, discoidal [12-14] and nanopolypods [15]. The biodistribution of drug carriers in a vascular network will depend on many parameters such as particle size, shape, and local flow conditions [16, 17]. For instance, several studies have demonstrated that particle binding is inversely correlated with shear rates $[18,19]$. The adhesion of nanocarriers also depends on targeting antibodies' affinity and surface density [20]. Moreover, junctions and bifurcations in microvasculature largely influence particle binding [21, 22].

The size of particle significantly influences drug targeting specificity and efficacy. For instance, targeting capabilities and uptake of small nano-particles (NP) are enhanced by their larger surface to volume ratio. On the other hand, bigger drug carriers can carry higher payloads when used in vascular targeting. Drug carriers bigger than $20 \mathrm{~nm}$ are eliminated from circulation mainly by liver, spleen and lymphatic nodes $[23,24]$ and particles larger than $\sim 500 \mathrm{~nm}$ generally have higher chance of depositing in capillaries [25]. Furthermore, carriers of radius under $100 \mathrm{~nm}$ are reported to migrate toward the vessel wall and interact with the endothelium more easily [26]. The deposition of spherical 
particles was shown to reduce monotonically as their diameter increase from $700 \mathrm{~nm}$ to $3 \mu \mathrm{m}$ [27]. Also, it is observed that 3-4 $\mu \mathrm{m}$ particles are more likely to be permanently trapped into the open circulation of the spleen[28].

Due to complex physiological conditions and bioethical regulations, it is very challenging to quantify the drug delivery in vivo. Therefore, most of experimental studies are carried out in flow channels [29, 30]. For example, Thomas et al. [31] performed an in-vitro study in a microfluidic chip mimicking microvasculature. They showed that particle binding density decrease with increased shear rates for $200 \mathrm{~nm}$ and $2 \mu \mathrm{m}$ particles and reported $10 \%$ higher binding at branching regions compared to straight sections. In that study, specificity in targeting is introduced by applying ligand-receptor chemistry on targeted region. Their biomimetic chip were coated with intercellular adhesion molecule 1 (ICAM-1) protein to study the influence of antibody density on binding.

Numerical models have been extensively used to study drug particle behavior in blood flow. Liu et al. [32] combined Monte Carlo and weighted histogram analysis method to study the nanocarrier binding affinities. Lee et al. [33] used immersed finite element method to simulate a nanoparticle focusing lens in a microfluidic channel. Gentile et al. [34] investigated the effect of vessel permeability and blood rheology on the transport of nanoparticles. Longest et al. [35] simulated the blood particle deposition process in a non-parallel flow. Decuzzi and Ferrari [36] studied the ligand-receptor binding dynamics of non-spherical particles and proposed a simple formula for binding probability incorporating several factors such as hemodynamic forces, receptor and ligand density. However, there is no generalized model which could link nano-scale adhesion dynamic to measurable total deposition densities. Such a model should be benchmarked with the existing experimental data and can serve as a predictive tool in drug deliver studies.

In this paper, a numerical approach is developed to study drug particle deposition in a branched micro-channel under various shear rates. This model captures the complex dynamics of carrier transport and binding as well as their effect on actual deposition rate. Direct comparison with our previous in-vitro testing results [31] is performed for particles of different sizes and surface chemistries. Our particulate approach helps capture phenomena which cannot be achieved by continuum models. In what follows, model geometry and parameters are first introduced. After discussing the complex correlation between binding probability and actual deposition, the effects of shear, antibody density, and geometry on particle binding are studied and discussed in detail.

\section{Computational model}

In our previous in-vitro experiments of NP binding [31], ligand-receptor chemistry is applied on a microfluidic platform. The substrate of the biomimetic chip is coated with intercellular adhesion molecule 1 (ICAM-1) protein with density of $121 \pm 12$ sites $/ \mu \mathrm{m}^{2}$. The fabrication process of microfluidic device as well as coating/characterization of particles with ICAM-1 and substrate with 
anti-ICAM-1 are described in our prior work [31] where the binding densities of $200 \mathrm{~nm}$ and $2 \mu \mathrm{m}$ particles were reported under various shear rates. Following the experimental conditions, we performed numerical simulations with mean inlet velocity of 0.05 to $0.4 \mathrm{~m} / \mathrm{s}$ which correspond to physiologically relevant shear rates of 200 to $1600 \mathrm{~s}^{-1}$ observed in the microvessels $[37,38]$. The kinematic viscosity of the buffer solution is $1 \mathrm{cP}$.

In this study, the flow in microfluidic channel is assumed to be laminar and Newtonian. The steady state flow field is solved using conventional discretized form of Navier-Stokes equation [16]. Trajectories of discrete phase particles are calculated by integrating the force balance on the particle using Velocity Verlet algorithm in a Lagrangian reference frame. The particle velocity can be expressed as:

$$
\frac{d u_{p}}{d t}=\frac{18 \mu}{\rho_{p} d_{p}^{2}}\left(\vec{u}-\vec{u}_{p}\right)+F_{b_{i}}
$$

where $\vec{u}$ is the fluid phase velocity, $\vec{u}_{p}$ is the particle velocity, $\rho_{p}$ is the density of the particle, $d_{p}$ is the particle diameter, $\mu$ is the viscosity of the fluid and $F_{b_{i}}$ is the Brownian force. The amplitudes of the Brownian force components are of the form

$F_{b_{i}}=\zeta_{i} \sqrt{\frac{\pi S_{0}}{\Delta t}} ; S_{0}=\frac{216 v k_{B} T}{\pi^{2} \rho d_{p}^{5}\left(\frac{\rho p}{\rho}\right)^{2}}$

where $\zeta_{i}$ are zero-mean, unit-variance-independent Gaussian random number, and $\Delta t$ is the calculation time step. $\mathrm{T}$ is the absolute temperature of the fluid, $v$ is the kinematic viscosity and $k_{B}$ is the Boltzmann constant. The detail description about how particles react as they hit the microchannel boundaries and how binding probability function integrated into model are discussed in the next section.

A setup similar to experiments is used in our computational model as shown in Fig 1a. The particle binding density is measured at the center section of the bottom channel surface. The width and height of channels are both $100 \mu \mathrm{m}$. The sampling regions of parent and daughter vessels are located far from inlet and bifurcation, respectively. The area which is considered as branching area starts from the point where straight channel bifurcate and ends at the daughter channels. 

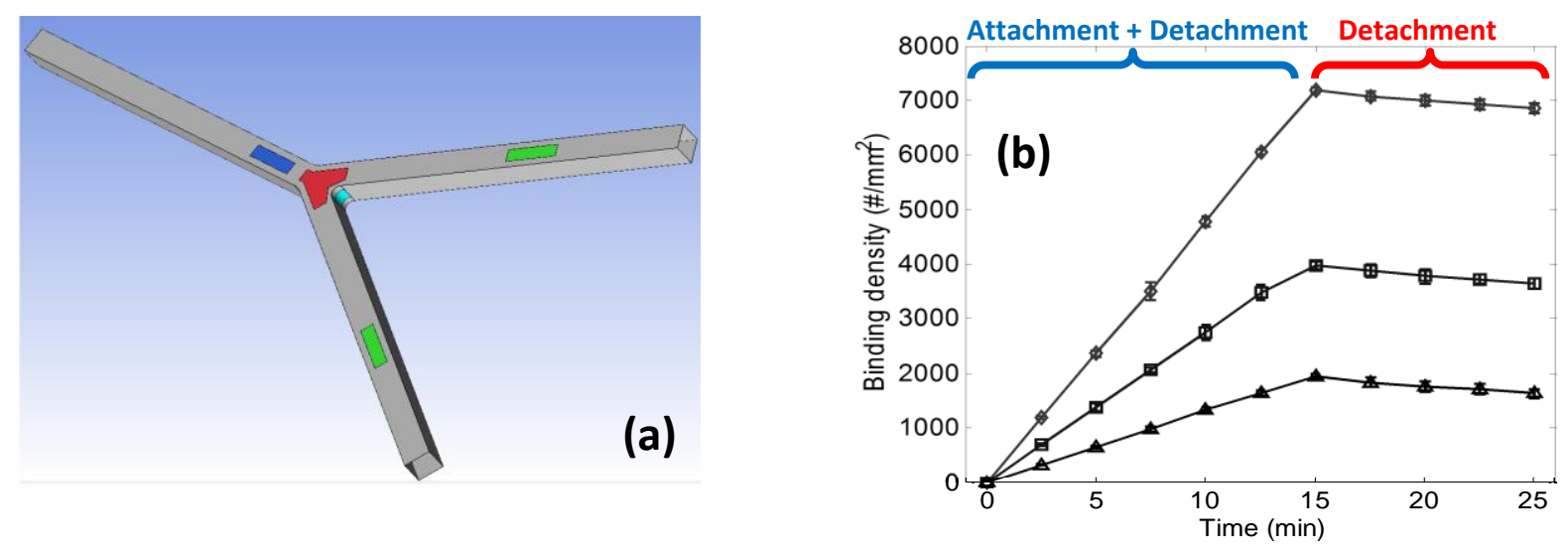

Fig 1. (a) Sampling regions: Dark blue, red, light blue and green color regions correspond to parent, branching, bifurcation and daughter sampling regions, respectively. (a) Time history of binding density for 200nm particles for three anti-ICAM-1 densities [31].

\section{Binding probability of particles}

Particles in flow field disperse and may come in contact with the treated substrate. The thermal diffusion of $200 \mathrm{~nm}$ particles are 10 times faster than that of $2 \mu \mathrm{m}$ particles since the diffusion coefficient is inversely related to the particles size according to Einstein theory of Brownian motion. Thus, 200nm particles will hit the boundaries 10 times more frequently if the diffusion is the only lateral force. Based on continuum formulation, the deposition rate at the wall is a linear function of diffusion coefficient for fully developed laminar flow in microchannel. Thus, 10 times larger binding rate are expected for $200 \mathrm{~nm}$ particles compared to $2 \mu \mathrm{m}$ particles. However, our experimental results [31] showed that 200nm particles have 2 to 9 times higher binding density depending on flow shear rate. Therefore, some particles may fail to bind or even dislodge during collision with vessel wall. It is observed that the attachment and detachment processes happen concurrently as shown in Fig $1 \mathrm{~b}$.

Continuum models use kinetic rate constants at boundaries for mimicking adhering and dislodging processes [29]. In our particulate numerical study, inspired from Decuzzi and Ferrari's work [36], attachment and detachment rates are replaced with an adhesion probability function. When the distance between particles center of mass and wall is less than particle's radius, adhesion probability function is called to determine the chance that particle has to permanently deposit on microchannel wall. By generating a random number between 0 and 1, the fate of particle is decided. Particles who fail to bind will be reflected with opposite velocity. However, these failed particles will still have the chance to hit the wall again and successfully adhere to treated microfluidic substrate downstream.

After releasing one million particles in a sample flow rate of $0.2 \mathrm{~mm}^{3} / \mathrm{s}$, the total number of particles deposited are recorded for a large range of adhesion probabilities as shown in Fig 2a. In contrary to our expectations, a considerable number of particles can still successfully bind when the chance of binding is as low as $0.1 \%$. It is observed that the correlation between binding probability and actual deposition rate is not linear. 
The binding probability function introduced by Decuzzi [36] is directly related to the strength of adhesion and is plotted out in Fig $2 \mathrm{~b}$ for spherical and non-spherical drug carriers. Since the binding process is stochastic in nature, their proposed probability function estimates the likelihood of having closed ligand-receptor bonds. Their binding probability varies within three orders of magnitude. For instance, one elliptical particle in low shear rate flow can have $30 \%$ chance of forming bond whereas another spherical particle with the same diameter may just have $0.3 \%$ odds of binding at slightly higher shear rate flow. This significant difference in binding probabilities may seem unrealistic at first, but based on what was just described, the overall binding rate might be 2 to 9 times bigger as observed in in-vitro experiments. In the following sections, we will discuss the effect of shear stress, particle diameter, anti-ICAM1 density, and channel geometry on particle binding.
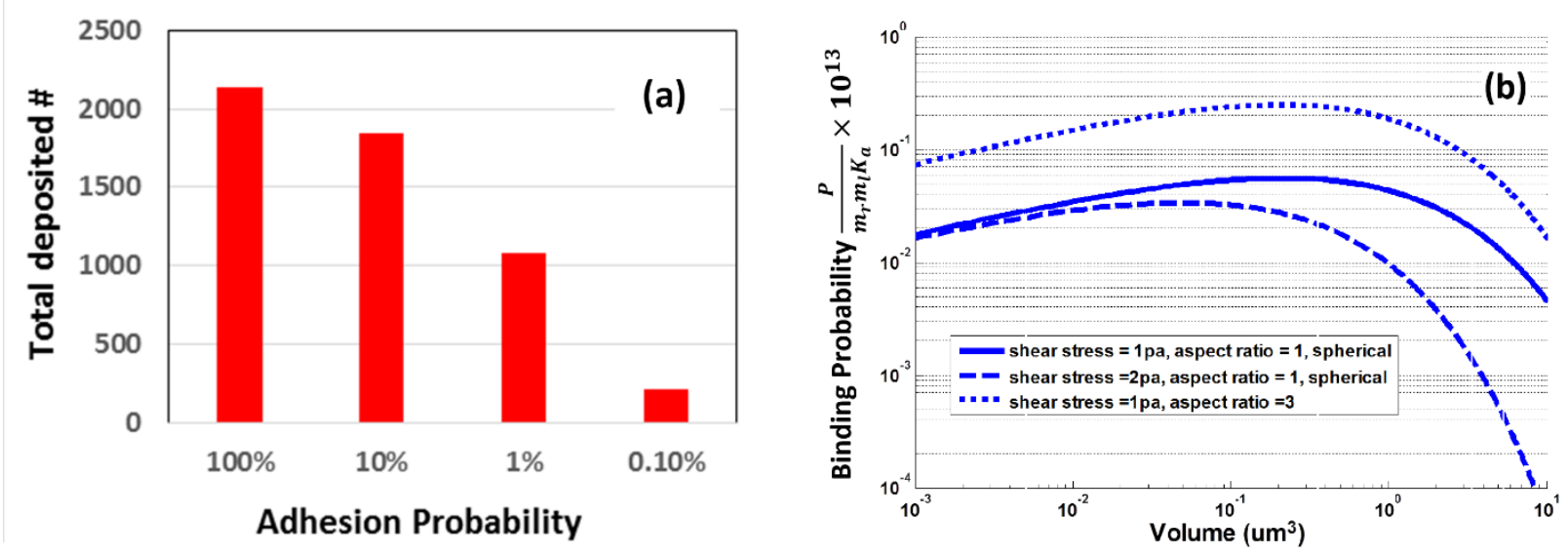

Fig 2. (a) Deposition rates for cases with various adhesion probabilities; (b) Binding probability versus particle volume for two different shear stresses and aspect ratios.

\section{Results and Discussion}

\section{Effect of shear stress and carrier diameter}

Piper et al. [39] mathematically evaluated the probability $\left(P_{0}\right)$ of a cell having no bond (detached). Using Taylor series expansion, the probability of having closed bonds can be derived as $P_{a}=1-$ $P_{0} \approx A_{c} m_{r} m_{l} K_{a}(f)$ where the constitutive equation for binding affinity, $K_{a}(f)$, is a function of dislodging force, $f$. Following this concept, Decuzzi and Ferrari [36] proposed that the ligands with particle surface density of $m_{l}$ and the receptors with substrate surface density of $m_{r}$ can stochastically form successful bonds at probability $P_{a}$ when particle hit the substrate. It can be expressed as

$P_{a}=m_{r} m_{l} K_{a}^{0} \pi r_{0}^{2} \exp \left[-\frac{\lambda d \mu S}{2 k_{B} T \mathrm{r}_{0}^{2} m_{r}}\left[6\left(d / 2+\delta_{e q}\right) F^{S}+2 \frac{d^{2}}{r_{0}} T^{S}\right]\right]$

$r_{0}=\frac{d^{2}}{2} \sqrt{\left(1-\left(1-\frac{2 *\left(h_{0}-\delta_{e q}\right)}{d}\right)^{2}\right)}$

where the coefficients $F^{S}$ and $T^{S}$ depend on aspect ratio [40]; $K_{a}^{0}$ is the association constant at zero load of the ligand-receptor pair; $r_{0}$ is the radius of interaction area between the particle and the 
substrate; $\lambda$ is a characteristic length of the ligand-receptor bond; $S$ is shear rate $\left(s^{-1}\right)$ and $d$ is particle diameter $(m)$. Other relevant parameters are listed in Table 1. Using Eq. 3, the adhesion probability of 200nm, $800 \mathrm{~nm}, 1.6 \mu \mathrm{m}$ and $2 \mu \mathrm{m}$ particles at different shear rates is plotted out in Fig 3.

Since it is very challenging to find the proper value for association constant at zero load $\left(K_{a}^{0}\right)$ from experiments, we have used one set of our experimental data (Fig. $4 \mathrm{~b}$ ) to tune $K_{a}^{0}$. Therefore, $K_{a}^{0}$ is tuned in a way that binding density of $200 \mathrm{~nm}$ particles in straight section of microchannel under low shear rates matches with experimental data. As shown in Fig 3, the adhesion probability of 200nm just slightly changes with shear rate and remains almost $1 \%$ under various physiologically relevant shear rates. We understood that utilizing constant adhesion probability of $1 \%$ for $200 \mathrm{~nm}$ particles under low shear flows we get a match between experiment and our model. In the following, it is shown that the extensive match with our other experimental results suggests that this tuned version of adhesion probability function can correctly predict adhesion dynamic as particles come in contact with microchannel wall.

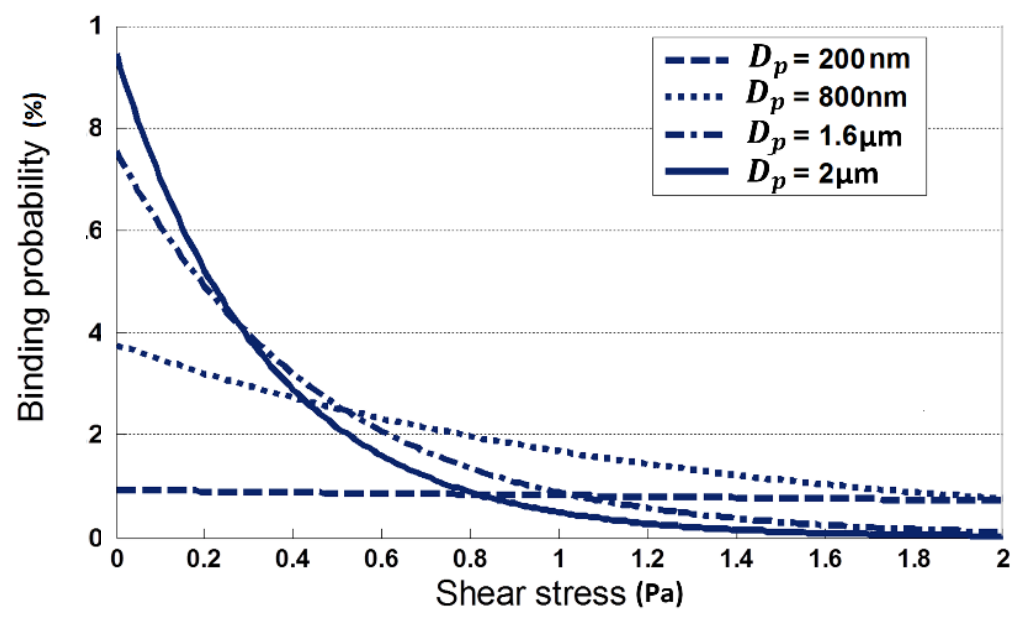

Fig 3. Binding probability for particles of different sizes versus shear stress.

Table 1. List of relevant parameters.

\begin{tabular}{|l|l|l|c|}
\hline & \multicolumn{1}{|c|}{ Parameters } & \multicolumn{1}{c|}{ Value } & Unit \\
\hline$\lambda$ & Ligand-receptor length & $10^{-10}$ & $\mathrm{~m}$ \\
\hline$k_{B} T$ & Boltzman thermal energy & $4.14 \times 10^{-21}$ & $\mathrm{~m}^{2} \cdot \mathrm{Kg}^{-2} \mathrm{~s}^{-2}$ \\
\hline$\mu$ & Kinematic viscosity & 1 & $c P$ \\
\hline$h_{0}$ & $\begin{array}{l}\text { Maximum separation } \\
\text { distance }\end{array}$ & $10^{-8}$ & $\mathrm{~m}$ \\
\hline$\delta_{e q}$ & $\begin{array}{l}\text { Equilibrium separation } \\
\text { distance }\end{array}$ & $5 \times 10^{-9}$ & $\mathrm{~m}$ \\
\hline
\end{tabular}

To study the influence of shear rate on binding density, 200nm particles are tracked under different flow rates. Two separate cases with adhesion probabilities of $100 \%$ and $1 \%$ are considered while their 
binding densities are calculated for branching and straight sections of microchannel as shown in Fig 4a-b. The results indicates that $1 \%$ adhesion probability shows best agreement with experimental results for $200 \mathrm{~nm}$ particles. On the other hand, it was mentioned that the adhesion probability of $2 \mu \mathrm{m}$ particles decreases exponentially with shear stress as shown in Fig 3. Cases with constant adhesion probability of $100 \%$ and $1 \%$ for $2 \mu \mathrm{m}$ particles are also considered for comparison purposes. It is observed that for $2 \mu \mathrm{m}$ particles a good agreement with experimental data can be found for the case where binding probability significantly decreases with shear stress as shown in Fig 4c-d.
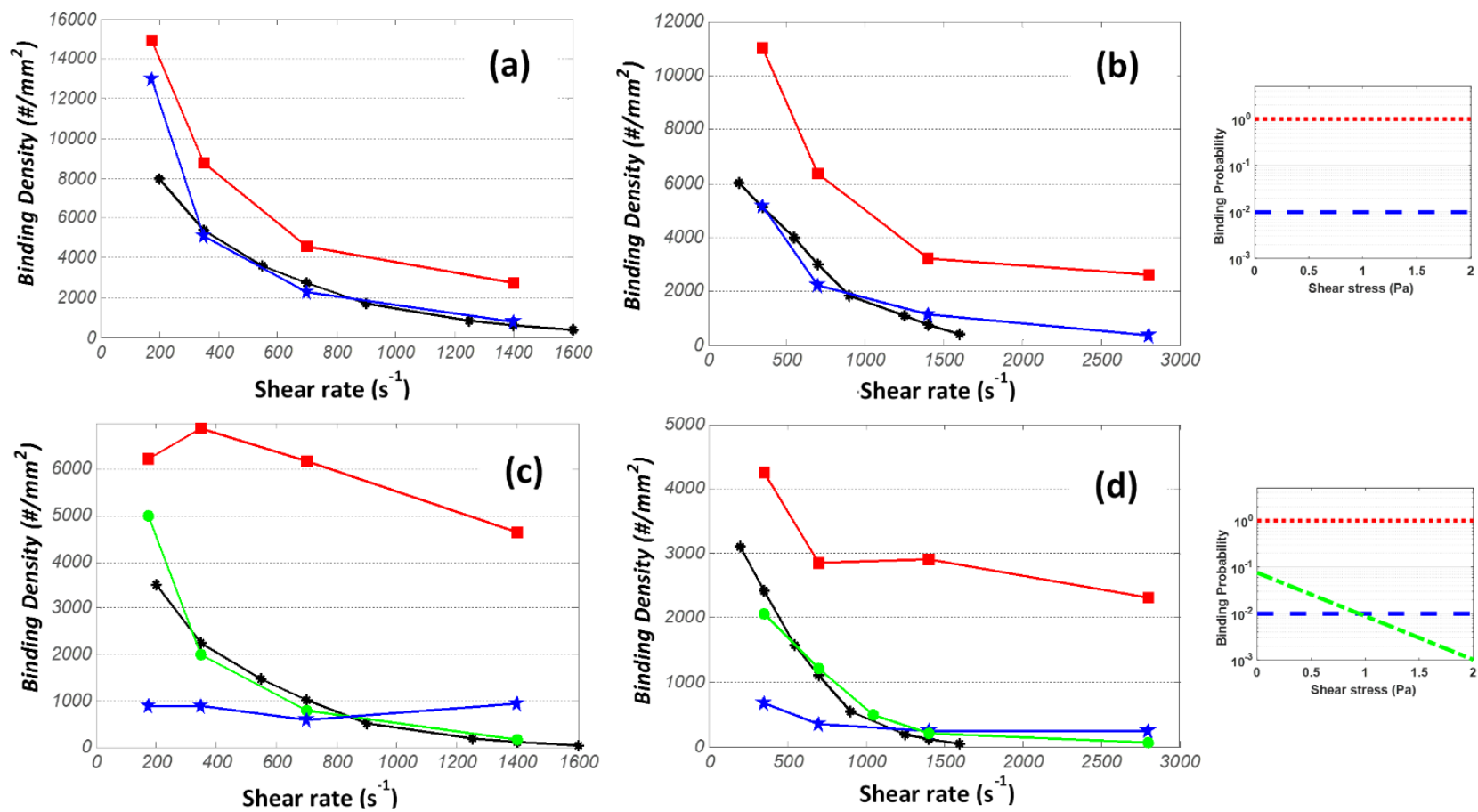

Fig 4. (a) Binding density (\#/mm²) of 200nm particles at branching sections of channels; (b) Binding density $\left(\# / \mathrm{mm}^{2}\right)$ of $200 \mathrm{~nm}$ particles at straight sections of channels; (c) Binding density $\left(\# / \mathrm{mm}^{2}\right)$ of $2 \mu \mathrm{m}$ particles at branching sections of channels; (d) Binding density $\left(\# / \mathrm{mm}^{2}\right)$ of $2 \mu \mathrm{m}$ particles at straight sections of channels; the solid black line represents experimental data [31]; Red and Blue represent cases with adhesion probabilities of $100 \%$ and $1 \%$, respectively; Green line represents exponentially decreasing adhesion probability of $2 \mu \mathrm{m}$ particles.

Using adhesion probability function described in Eq. 3, it is shown that our numerical results agrees well with experimental data. In other word, it suggests that the complex attachment and detachment processes can be simply replaced by an adhesion probability function. It is also observed that at low shear rates, the adhesion probability of small particles is not $100 \%$ because their smaller contact area considerably lowers their chance of binding. As illustrated in Fig 3, the adhesion probability of big 
particles is higher than smaller ones at lower shear rates, but it does not necessarily means that their actual deposition rate is also higher because their lower mobility significantly reduces their total number of contact with wall. Overall, it is shown that the tuned version of probability function can nicely model the complicated attachment and detachment process of decorated carriers to/from treated substrate.

\section{Influence of anti-ICAM density on particle binding}

An extensive parametric analysis is performed on our previous experimental study [31]. Our invitro tests in microfluidic chip were carried out for particles coated with anti-ICAM surface densities of $1905,1257,1199,625$ and $272\left(\# / \mu m^{2}\right)$. The binding density profiles were plotted out for $200 \mathrm{~nm}$ and $2 \mu \mathrm{m}$ particles under $200,550,900,1250$ and $1600\left(\mathrm{~s}^{-1}\right)$ shear rates. For facilitating the comparison, binding densities are normalized with maximum binding at $1905\left(\frac{\#}{\mu m^{2}}\right)$ anti-ICAM density for any given particle size and shear rate. Thus, the normalized binding density data points in Fig 5a only demonstrate the effect of anti-ICAM density on binding mechanics. Anti-ICAM density is also normalized with $m_{r 0}=1905\left(\frac{\#}{\mu m^{2}}\right)$. Fitting the experimental data points, a simple linear relationship is found between deposition rate and antibody density as shown in Fig 5a. It should be mentioned that the current analysis is done for particles flowing in pure buffer solution. Performing similar procedure for RBC flow cases, the same 1:1 relationship is also obtained although binding rate is considerably higher due presence of RBCs. Overall, it is observed that the binding linearly increases with anti-ICAM density regardless of flow dynamics.

Previous theoretical studies [39] suggested that antibody density, $m_{l}$, is a coefficient in probability formula as shown in Eq. 2. We performed numerical simulations by assuming linear variation of probability function with antibody density. Similar normalization procedures are also carried out here. As observed in Fig 5b, our numerical simulations nicely predict aforementioned 1:1 relationship and match with the experimental findings for anti-ICAM densities lower than $1260\left(\frac{\#}{\mu m^{2}}\right)$. Moreover, it is observed that binding rate will saturate when the substrate is coated with higher anti-body densities [31]. 

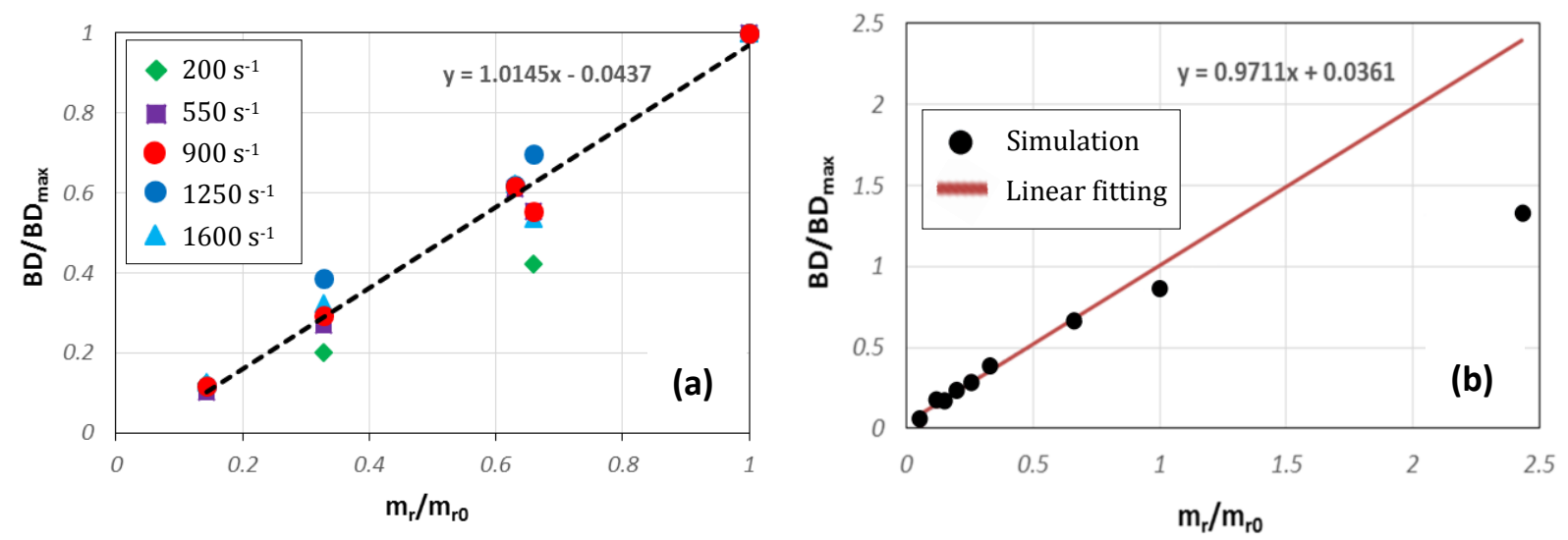

Fig 5. Normalized binding density (BD) profiles versus dimensionless antibody density; obtained from (a) experiments [31] and (b) simulation; In (a), colored dots represents different shear rates under which experiments are performed and dashed line represent linear fitting of these data; In (b), solid red line represent linear fitting for antibody density lower than $1260\left(\frac{\#}{\mu m^{2}}\right)$.

\section{Particle concentration distribution}

To calculate particle concentration distribution, drug carriers are tracked within steady flow and their locations are recorded as they pass through various cross sections of channel. This information can be translated into concentration profiles, red solid line, at different cross sections as shown in Fig 6. For better comparison, the shear stress is chosen as $0.8 \mathrm{~Pa}$ inducing similar adhesion probability of $1 \%$ for $200 \mathrm{~nm}$ and $2 \mu \mathrm{m}$ particles as shown in Fig 3. Blue dashed line in Fig 6 demonstrates the average concentration and the solid green line represents the concentration distribution when the adhesion probability is $100 \%$. The latter can also be derived by solution of conventional convection-transport equation since the process is fully diffusion limited and particles bind as soon as they hit the wall $\left(C_{w a l l}=0\right)$. It should also be mentioned that the concentration profile for $100 \%$ probability case will be fully developed into a parabolic shape further downstream.

Our initial thought was that the bulk concentration at wall for $1 \%$ binding probability cases should be higher because numerous particles fail to bind and therefore are reflected due to small binding probability. In contrary to our intuitions, the drug carrier concentration on the wall is zero for both $200 \mathrm{~nm}$ and $2 \mu \mathrm{m}$ cases and the reflected particles accumulate slightly further away from the treated surface where two spikes appear in concentration profiles. These spikes will not disappear downstream since it is an intrinsic characteristic of the reflecting boundary as shown in Fig 6b-c. Moreover, the concentration distribution profile is also plotted out for horizontal cross section of microchannel in Fig 6d where its shape in daughter branch suggests that the geometry is also an influential factor in local binding rates. 

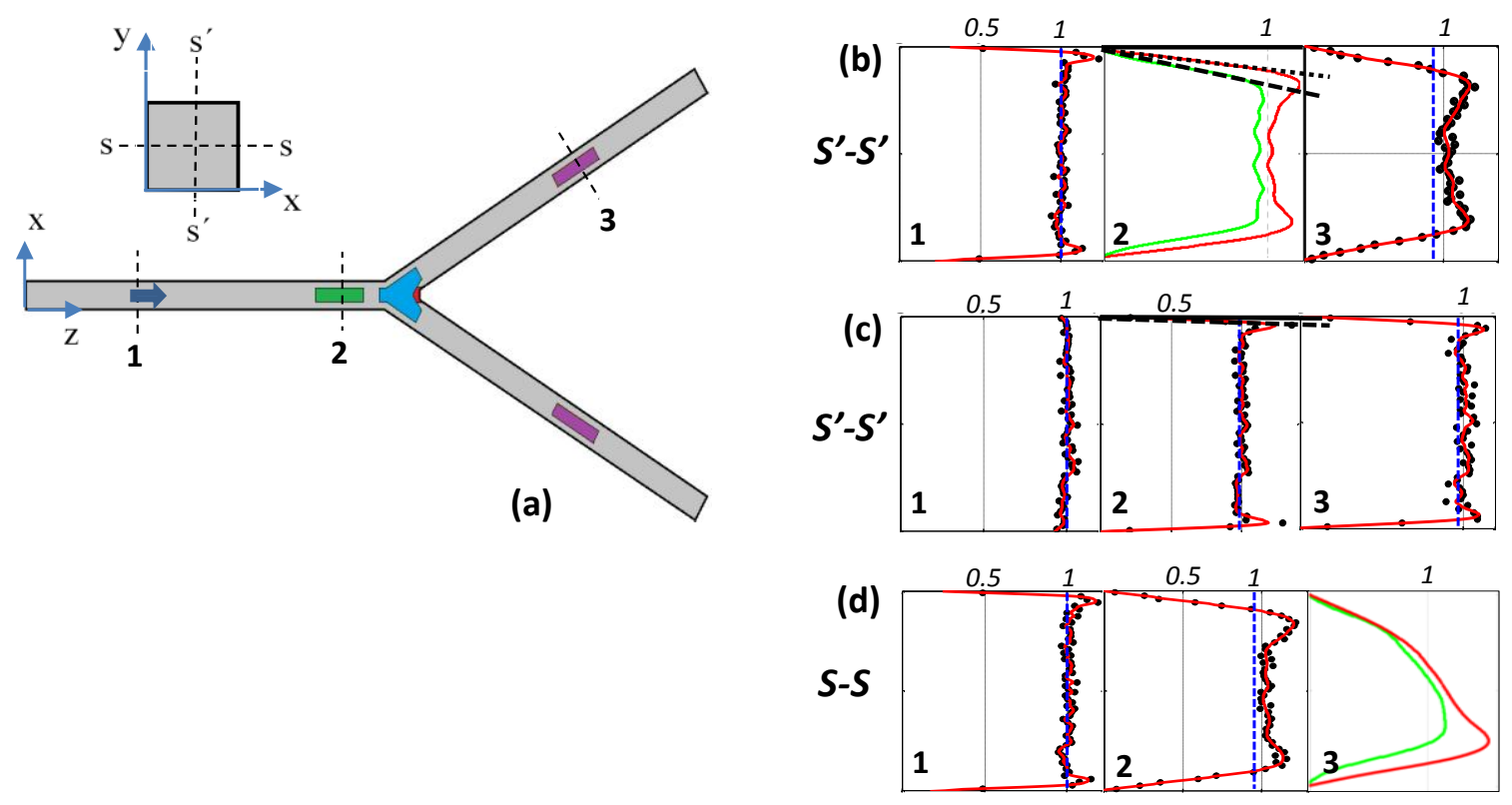

Fig 6. (a) The top and side views of microfluidic channel; Normalized concentration profiles $\left(C_{w} / C_{0}\right)$ in $\mathrm{S}^{\prime}-\mathrm{S}$ ' cross sections of micro-channel for (b) $200 \mathrm{~nm}$ and (c) $2 \mu \mathrm{m}$ particles; (d) Normalized concentration profiles $\left(C_{w} / C_{0}\right)$ in S-S cross sections of micro-channel for 200nm particles; Red solid lines represent fitted concentration data points; Blue dashed lines represent average concentration; Green lines are concentration distribution when binding probability is $100 \%$.

In continuum models [29], attachment and detachment rates are used to implement adhering and dislodging dynamics. The boundary condition in continuum approach can be expressed as $\left.D_{\text {diff }} \frac{\partial C_{w}}{\partial x}\right|_{x= \pm H / 2}=\frac{\partial C_{s}}{\partial t}=k_{a} C_{w}-k_{d} C_{s}$

where $C_{w}\left(\frac{\#}{\text { area }}\right)$ is the unbound particle concentration at surface; $C_{s}\left(\frac{\#}{\text { area }}\right)$ is the bound particle density; $k_{a}$ and $k_{d}$ are attachment and detachment kinetic rates, respectively. Our particulate simulations showed that the bulk concentration of unbound particle on substrate is zero while continuum model assumes nonzero bulk concentration in order to calculate wall attachment rate. Furthermore, adhesion rate is calculated by radial concentration gradient at the wall in continuum model. As shown in Fig 2a the binding rate of $100 \%$ case is higher than $1 \%$ case. Thus, continuum model would suggest higher wall gradient for $100 \%$ case while the comparison of green and red profiles in Fig 6a reveals an opposite scenario. Higher concentration gradient in $1 \%$ case can also be simply explained by the reflecting characteristics of boundaries. These two discussed differences between our method and continuum model originate from particulate nature of our modelling approach. Although continuum model assumptions are still valid, our particulate approach can capture more detail in particle's dynamics in microvessels.

Attachment and detachment are time dependent processes because binding rate may decrease by time when there are no more accessible ICAM-1 receptors on the channel wall. According to our in-vitro 
tests [31], the substrate binding density hits a saturation limit when the dispersion rate is considerably enhanced in the presence of RBCs. Our tests [31] indicated that the saturate binding density of $2 \mu \mathrm{m}$ particles at low shear rate RBC flow is approximately $20 \times 10^{3}\left(\# / \mu \mathrm{m}^{2}\right)$ while the highest binding density observed in buffer solution is $3 \times 10^{3}\left(\# / \mu \mathrm{m}^{2}\right)$. Since our goal in the current study is to simulate low deposition densities, binding process is assumed to be independent of time. Nevertheless, saturation by time can also be modelled by our particulate approach. The simplest way is to implement bound particle density, $C_{s}$, into adhesion probability function in order to locally manipulate binding chance as more particles deposit.

\section{Effect of 3-D channel geometry}

Bound particles at vertical bifurcating section cannot be easily counted by confocal microscope due to the limited z stack image resolution. Our numerical approach can provide a more convenient tool for studying particle deposition on such surfaces as shown in Fig 7a. The binding densities of 200nm and $2 \mu \mathrm{m}$ particles in two sampling regions are plotted out in Fig $7 \mathrm{~b}$. Although higher number of particles hit the bifurcating region, it is observed that the binding density is lower compared to branching region. As shown in Fig 7a, shear stress increases locally at the bifurcating region. Thus, higher shear rates in this region lower the chance of binding and dislodged particles will be quickly washed away from substrate. Furthermore, in lower flow rates, the adhesion probability of $2 \mu \mathrm{m}$ particles is significantly higher than 200nm particles, as shown in Fig $7 \mathrm{~b}$, while its binding density is almost 3 times lower, see Fig 3. The reason is that the total number of particle-substrate contacts is highly dependent on dispersion rate. Thus, both adhesion dynamic and particle transport are equally influential in the drug delivery to microvasculature.
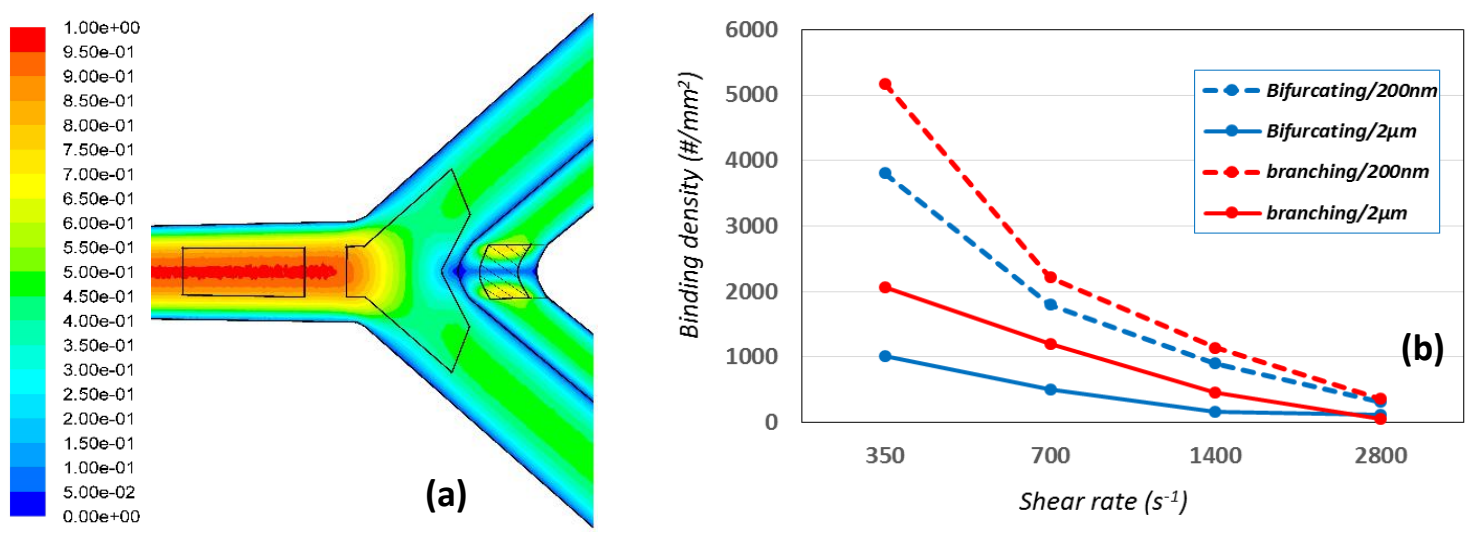

Fig 7 (a) Shear stress (Pa) distribution on microchannel wall; (b) binding density in bifurcating and branching sampling regions for $200 \mathrm{~nm}$ and $2 \mu \mathrm{m}$ particles.

\section{Conclusion}

In this study, we developed a particulate binding model and benchmarked it with our previous in-vitro test results. This simple, yet efficient numerical approach would help us predict particle delivery 
process. In our model, the adhesion probability function includes both adhering and dislodging processes. The nonlinear relationship between binding probability and actual deposition showed that even at low probabilities, considerable number of particles can still successfully adhere to the microchannel wall. Furthermore, constant and exponentially decreasing probability for $200 \mathrm{~nm}$ and $2 \mu \mathrm{m}$ particles are proven to best match experimental results. Our parametric study demonstrated that anti-ICAM density linearly increases deposition rate up to a certain threshold when it reaches its saturation limit. It is also observed that locally elevated shear stress at bifurcating region decreases deposition rate. Moreover, it is shown that our particulate approach captured more details in binding dynamic close to walls compared to continuum models. For instance, concentration distribution profiles at different sections of microfluidic channel showed that bulk concentration and its gradient do not follow continuum model assumptions. Overall, our benchmarked theoretical approach is an efficient tool to study drug particle delivery in microvasculature.

\section{ACKNOWLEDGMENT}

The authors acknowledge the supports of this work from National Science Foundation (NSF) CAREER grant CBET-0955214, NSF CBET-1067502, and National Institute of Health (NIH) grant EB015105.

\section{Reference}

1. Chauvierre, C., et al., Novel polysaccharide-decorated poly (isobutyl cyanoacrylate) nanoparticles. Pharmaceutical research, 2003. 20(11): p. 1786-1793.

2. Farokhzad, O.C. and R. Langer, Nanomedicine: developing smarter therapeutic and diagnostic modalities. Advanced drug delivery reviews, 2006. 58(14): p. 1456-1459.

3. Nasongkla, N., et al., Multifunctional polymeric micelles as cancer-targeted, MRIultrasensitive drug delivery systems. Nano letters, 2006. 6(11): p. 2427-2430.

4. Roney, C., et al., Targeted nanoparticles for drug delivery through the blood-brain barrier for Alzheimer's disease. Journal of Controlled Release, 2005. 108(2): p. 193-214.

5. Zhou, Y., et al., Mechanical Properties of Nanoworm Assembled by DNA and Nanoparticle Conjugates. Journal of Nanoscience and Nanotechnology, 2016. 16(6): p. 5447-5456.

6. Allen, T.M. and P.R. Cullis, Drug delivery systems: entering the mainstream. Science, 2004. 303(5665): p. 1818-1822.

7. Duncan, R., Polymer conjugates as anticancer nanomedicines. Nature Reviews Cancer, 2006. 6(9): p. 688-701.

8. Hirsch, L.R., et al., Nanoshell-mediated near-infrared thermal therapy of tumors under magnetic resonance guidance. Proceedings of the National Academy of Sciences, 2003. 100(23): p. 13549-13554.

9. Peer, D., et al., Nanocarriers as an emerging platform for cancer therapy. Nature nanotechnology, 2007. 2(12): p. 751-760. 
10. Tasciotti, E., et al., Mesoporous silicon particles as a multistage delivery system for imaging and therapeutic applications. Nature nanotechnology, 2008. 3(3): p. 151-157.

11. Slowing, I.I., et al., Mesoporous silica nanoparticles as controlled release drug delivery and gene transfection carriers. Advanced drug delivery reviews, 2008. 60(11): p. 1278-1288.

12. $\mathrm{Xu}, \mathrm{Q}$., et al., Preparation of monodisperse biodegradable polymer microparticles using a microfluidic flow - focusing device for controlled drug delivery. Small, 2009. 5(13): p. 15751581.

13. Rolland, J.P., et al., Direct fabrication and harvesting of monodisperse, shape-specific nanobiomaterials. Journal of the American Chemical Society, 2005. 127(28): p. 10096-10100.

14. Champion, J.A., Y.K. Katare, and S. Mitragotri, Particle shape: a new design parameter for micro-and nanoscale drug delivery carriers. Journal of Controlled Release, 2007. 121(1): p. 39.

15. Ren, J. and R.D. Tilley, Shape - Controlled Growth of Platinum Nanoparticles. Small, 2007. 3(9): p. 1508-1512.

16. Sohrabi, S., et al., Numerical Simulation of Particle Transport and Deposition in the Pulmonary Vasculature. Journal of biomechanical engineering, 2014. 136(12): p. 121010.

17. Tan, J., et al., Characterization of Nanoparticle Dispersion in Red Blood Cell Suspension by the Lattice Boltzmann-Immersed Boundary Method. Nanomaterials, 2016. 6(2): p. 30.

18. Lin, A., et al., Shear-regulated uptake of nanoparticles by endothelial cells and development of endothelial-targeting nanoparticles. Journal of Biomedical Materials Research Part A, 2010. 93A(3): p. 833-842.

19. Blackwell, J.E., et al., Ligand coated nanosphere adhesion to E-and P-selectin under static and flow conditions. Ann Biomed Eng, 2001. 29(6): p. 523-33.

20. Muzykantov, V.R., R. Radhakrishnan, and D.M. Eckmann, Dynamic factors controlling targeting nanocarriers to vascular endothelium. Current drug metabolism, 2012. 13(1): p. 70.

21. Prabhakarpandian, B., et al., Synthetic microvascular networks for quantitative analysis of particle adhesion. Biomedical microdevices, 2008. 10(4): p. 585-595.

22. Tousi, N., et al., Preferential adhesion of leukocytes near bifurcations is endothelium independent. Microvascular research, 2010. 80(3): p. 384-388.

23. Leucuta, S.E., Systemic and biophase bioavailability and pharmacokinetics of nanoparticulate drug delivery systems. Curr Drug Deliv, 2013. 10(2): p. 208-240.

24. Fraley, R. and D. Papahadjopoulos, Liposomes: the development of a new carrier system for introducing nucleic acid into plant and animal cells. Curr Top Microbiol Immunol, 1982. 96: p. 171-91.

25. Shuvaev, V.V., et al., Modulation of endothelial targeting by size of antibody-antioxidant enzyme conjugates. Journal of Controlled Release, 2011. 149(3): p. 236-241.

26. Decuzzi, P., et al., A theoretical model for the margination of particles within blood vessels. Ann Biomed Eng, 2005. 33(2): p. 179-90.

27. Decuzzi, P., et al., Size and shape effects in the biodistribution of intravascularly injected particles. Journal of Controlled Release, 2010. 141(3): p. 320-327.

28. Chen, L.-T., Microcirculation of the spleen: and open or closed circulation? Science, 1978. 201(4351): p. 157-159.

29. Haun, J.B. and D.A. Hammer, Quantifying nanoparticle adhesion mediated by specific molecular interactions. Langmuir, 2008. 24(16): p. 8821-8832.

30. Kona, S., et al., Biodegradable nanoparticles mimicking platelet binding as a targeted and controlled drug delivery system. International journal of pharmaceutics, 2012. 423(2): p. 516524.

31. Thomas, A., J. Tan, and Y. Liu, Characterization of nanoparticle delivery in microcirculation using a microfluidic device. Microvascular research, 2014. 94: p. 17-27. 
32. Liu, J., et al., Computational model for nanocarrier binding to endothelium validated using in vivo, in vitro, and atomic force microscopy experiments. Proceedings of the National Academy of Sciences, 2010. 107(38): p. 16530-16535.

33. Lee, S.-Y., M. Ferrari, and P. Decuzzi, Design of bio-mimetic particles with enhanced vascular interaction. Journal of biomechanics, 2009. 42(12): p. 1885-1890.

34. Gentile, F., et al., The effect of shape on the margination dynamics of non-neutrally buoyant particles in two-dimensional shear flows. Journal of biomechanics, 2008. 41(10): p. 23122318.

35. Longest, P.W. and C. Kleinstreuer, Comparison of blood particle deposition models for nonparallel flow domains. Journal of Biomechanics, 2003. 36(3): p. 421-430.

36. Decuzzi, P. and M. Ferrari, The adhesive strength of non-spherical particles mediated by specific interactions. Biomaterials, 2006. 27(30): p. 5307-5314.

37. Nagaoka, T. and A. Yoshida, Noninvasive Evaluation of Wall Shear Stress on Retinal Microcirculation in Humans. Investigative Ophthalmology \& Visual Science, 2006. 47(3): p. 1113-1119.

38. Patrizia Nigro, J.-i.A., and Bradford C. Berk., Flow Shear Stress and Atherosclerosis: A Matter of Site Specificity. Antioxidants \& Redox Signaling, 2011. 15(5): p. 1405-1414.

39. Piper, J.W., R.A. Swerlick, and C. Zhu, Determining force dependence of two-dimensional receptor-ligand binding affinity by centrifugation. Biophysical journal, 1998. 74(1): p. 492513.

40. Goldman, A., R. Cox, and H. Brenner, Slow viscous motion of a sphere parallel to a plane wall-Il Couette flow. Chemical Engineering Science, 1967. 22(4): p. 653-660. 